\title{
Oto-acoustic emissions and brainstem evoked response audiometry in patients of tinnitus with normal hearing
}

Shubham Dadoo Rohit Sharma* Vineet Sharma

\begin{abstract}
Introduction: Tinnitus is defined as the perception of sound that results completely from activity within the nervous system without any corresponding mechanical, vibratory activity within the cochlea, and not related to external stimulation of any kind. It disrupts the daily life of 1 out of every 200 adults. The source of tinnitus generation is not limited to the peripheral auditory system. However, there are abnormalities seen in BERA in tinnitus patients depicting auditory pathway involvement. Oto-acoustic emissions are mechanical vibrations generated in the cochlea, which are evaluated by TEOAE and DPOAE whereas BERA evaluates both cochlea and brainstem auditory pathway for any conduction abnormalities. The aim of the study is to analyze the changes in OAE and BERA in patients suffering from tinnitus with normal hearing, which may help us to understand the patho-physiology of tinnitus.
\end{abstract}

Material and methods: This is a prospective study conducted in a tertiary care hospital in Northern India between $1^{\text {st }}$ December 2015 to $31^{\text {st }}$ July 2017. All patients of tinnitus with normal hearing were included in the study group, whereas Individuals with normal hearing with no other ear complaints were included in control group. Total 160 Ears were evaluated with 80 ears in both study and control group each. Patients with PTA $>25 \mathrm{~dB}$, age $>55$ years or any chronic medical illness were excluded from the study.

Results: 80 individuals (46 Males and 34 Females) were divided into study and control Group (80 Ears each). Tinnitus was bilateral in 28 subjects (53.84\%) and unilateral in 24 subjects (46.16\%). Both control and study group showed significant difference in TEOAE and DPOAE study. In TEOAE, $8(10 \%)$ ears in control group and 30 ears $(37.5 \%)$ in study group showed test result as REFER whereas in DPOAE 10 (12.5\%) ears in control group and 35 (43.8\%) ears showed test result as REFER. All these result were statistically significant. In BERA the latency of wave I was significantly prolonged in study group as compared to control group, while difference between all other parameters between the two groups was insignificant.

Conclusion: There were various significant abnormalities seen in parameters of Oto-Acoustic Emissions (OAE) and Brainstem Evoked Response Audiometry (BERA). So these tests should be included in the test battery for the screening of patients complaining of tinnitus even with normal hearing.

Keywords: Oto acoustic emission, brainstem evoked response audiometry, tinnitus, normal hearing.

Department of Otorhinolaryngology, Head \& Neck Surgery, Shri Ram Murti Institute of Medical Sciences, Bareilly, UP, India

* Send correspondence to:

Rohit Sharma

Department of Otorhinolaryngology, Head \& Neck Surgery, Shri Ram Murti Institute of Medical Sciences, Bareilly, UP, India, E-mail: rohitsharma.dr@gmail.com

Phone: +9997449995

Paper submitted to the ITJ-EM (Editorial Manager System) on January 21, 2019; and accepted on April 15, 2019. 


\section{INTRODUCTION}

Tinnitus is defined as the perception of sound that results completely from activity within the nervous system without any corresponding mechanical, vibratory activity within the cochlea, and not related to external stimulation of any kind $^{1}$. Tinnitus disrupts the daily life of 1 out of every 200 adults. $85-96 \%$ of patients with tinnitus present with some levels of hearing loss whereas only $8-10 \%$ have normal hearing ${ }^{2}$. The source of tinnitus generation is not limited to the peripheral auditory system. However, there are abnormalities seen in BERA in tinnitus patients depicting auditory pathway involvement ${ }^{3}$. Otoacoustic emissions are mechanical vibrations generated in the cochlea, that are transmitted by the endolymphatic fluid in the cochlea and by the middle ear to the outer ear canal where they can be recorded as generally weak sounds with a microphone ${ }^{4}$. Auditory Brainstem Response (ABR) can be used in evaluating tinnitus patients for a number of reasons, including its subjectivity in evaluating the cochlea and the brainstem auditory pathways ${ }^{5}$. It examines the synchronous discharge of fibers in the auditory pathway and identifies the presence of abnormal neuronal activity. ABR may assist in the differentiation of central vs. peripheral tinnitus 6 . Tinnitus is a common and persistent symptom. The pathogenesis and site of origin have yet to be clearly established. Tinnitus is often a feature of primary ear disease usually associated with hearing loss, but it may also occur in patients with normal hearing. Our study focused on the latter group. The lack of scientific evidence to prove that tinnitus arises from cochlear damage in normal-hearing patients has encouraged us to investigate whether patients with tinnitus show changes in the auditory pathways. The aim of the study is to analyze the changes in OAE and BERA in patients of tinnitus with normal hearing, which may help us to understand the patho-physiology of tinnitus (Figure 1).

\section{MATERIALS AND METHODS}

The study was conducted in the Department of ENT and Head and Neck Surgery at SRMS Institute of Medical Sciences, Bareilly in patients presenting to ENT OPD with tinnitus from 1st December 2015 to 31st July 2017, after the approval by the Research/Ethics Committee. Individual Ear was considered as the study unit, as some patients had unilateral symptom. All the ears of the patients suffering from tinnitus with normal hearing were included in the study group, whereas Individuals with normal hearing with no other ear complaints were included in control group. Thus a total of 160 Ears were included in the study, both Study Group and Control Group contained 80 Ears each. All the patients with history of Tinnitus underwent a complete ENT evaluation including tuning fork test and Pure Tone Audiometry (PTA) (Figure 1). Patients with normal otoscopic findings and pure tone average below $25 \mathrm{~dB}$ formed the study group. Individuals with no complaints regarding ENT, with normal otoscopic findings and pure tone average below $25 \mathrm{~dB}$ were included in control group. Patients with hearing Impairment, pure tone average $>25 \mathrm{~dB}$, Age $\geq 55$ years, any external or middle ear disease like wax, otomycosis, ear discharge, perforation in tympanic membrane, Otitis Media with effusion, any history of intake of ototoxic drugs or Anti-tubercular drugs, associated psychiatric illness, noise exposure, acoustic trauma or any chronic medical illness like diabetes mellitus, hypertension or neuropathy were excluded from the study. Patients of both study and control group underwent OAE and BERA.

Both OAE and BERA were performed in a sound treated room of the Neuro-otology lab in supine position by a qualified audiologist, using Neurosoft (Russia) system with Neuro-Audio version 2010. The system was calibrated on daily basis using the calibration mode in the software. The

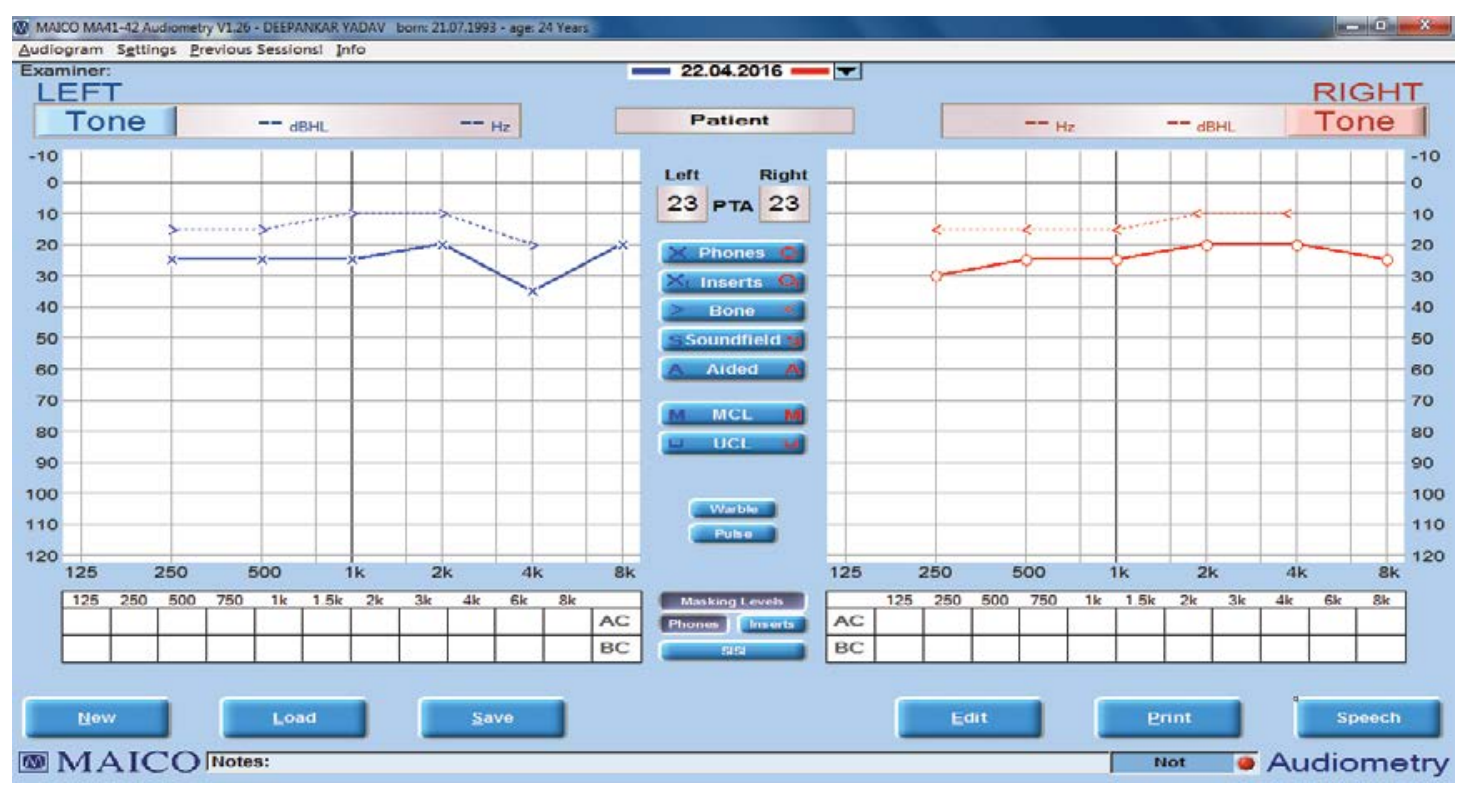

Figure 1. Figure showing PTA 


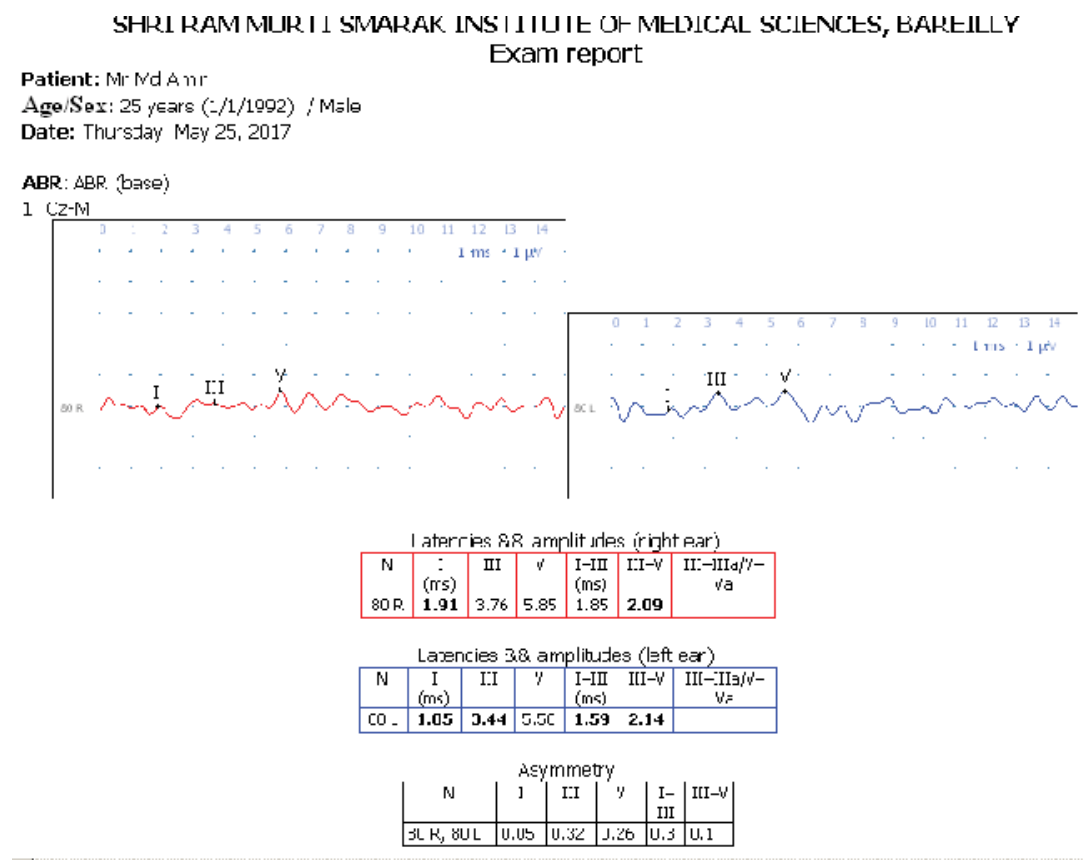

Figure 2. Brainstem Evoked Response Audiometry Tracing

first test performed was distortion product Oto-acoustic emissions. The probe seal and any extrinsic noise levels were checked by the software and were confirmed. The frequencies tested were from $2-6 \mathrm{kHz}$. The graph was plotted simultaneously along with the acquisition of data. During the measurement, two pure tone stimuli (f1 and f2), where $\mathrm{f2}$ is higher than f1 were presented with $\mathrm{f} 1 / \mathrm{f} 2$ ratio at approximately 1.22 (range 1.21 to 1.23 ) to obtain a robust DPOAE response. The $\mathrm{f} 2$ frequencies were tested in a 2 point per octave manner, from $2 \mathrm{kHz}$ to $6 \mathrm{kHz}$. Two stimuli were presented at an asymmetrical intensity level of $L 1=65 \mathrm{~dB}$ SPL and the second intensity, $L 2=55 \mathrm{~dB}$ SPL (such that L1 $>$ L2) (Figure 3). In the second stage of the first test, TEOAE were measured by providing stimuli of 80-85 dB SPL. The stimulation rate was less than 60 stimuli per second. TEOAEs were recorded in the time domain over approximately 20 milliseconds. The graph was plotted simultaneously along with the acquisition of data. The results were interpreted as 'PASS' or 'REFER'. PASS means that the patient's outer hair cell functioning in each ear is normal at the time of testing whereas REFER is suggestive of outer hair cell dysfunction (Figure 4).

After OAE, BERA was performed in the same sitting. The active electrode was placed over the vertex of the head and the reference electrode was placed over

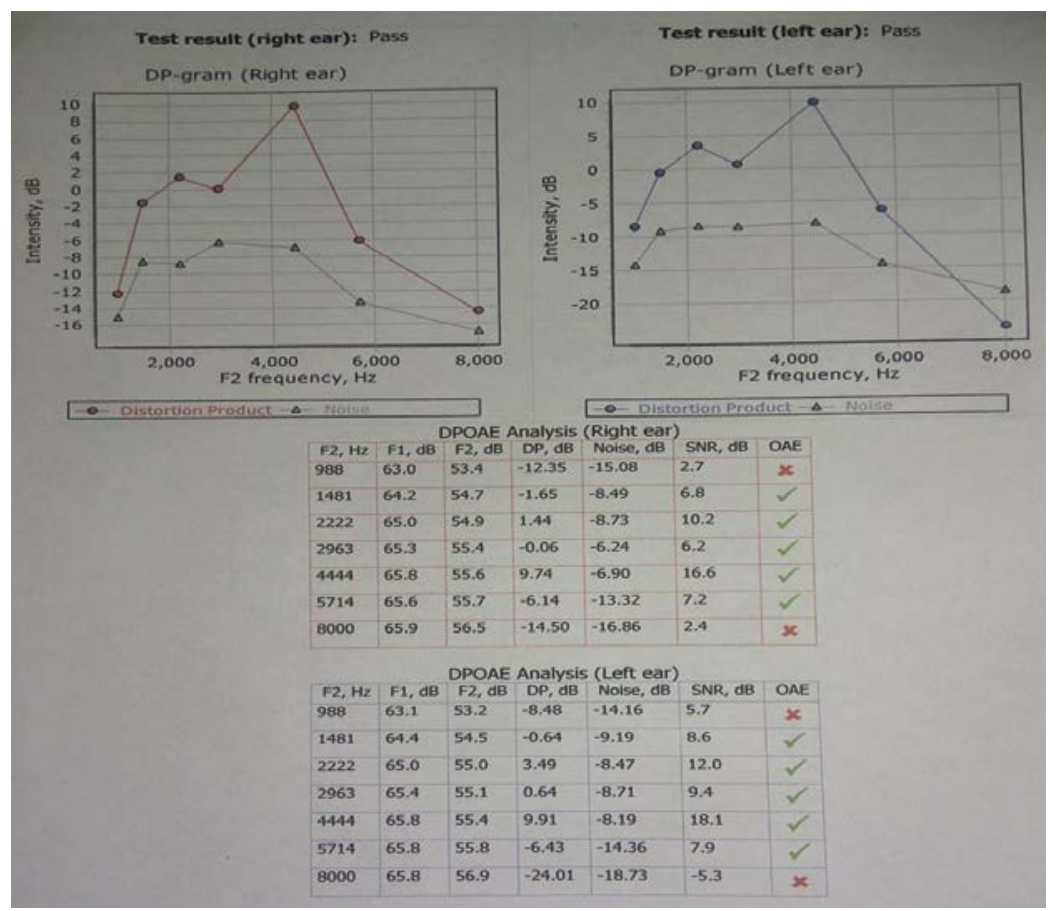

Figure 3. Figure showing the DPOAE Tracings 
mastoid prominences of both sides. One more ground electrode was placed over the forehead. Electrodes were attached using skin prep gel (Nu Prep, USA) in order to maintain impedance below 3000 ohms. Stimulus rate was kept between 10-30 stimuli per second with repetitive click stimulus of $80 \mathrm{~dB}$. Each ear was tested separately while the opposite ear was masked. The impulses were recorded, which contained a series of peaks and troughs. The positive peaks were referred by the Roman numerals I-VII based on their location of origin. These peaks occur in most readable form in response to click stimuli over a period of 1-10 milliseconds after the stimulus in normal hearing adults. The evaluated parameters included absolute latencies of wave I, III and V and Inter-peak latency between I-III, III-V, I-V.

\section{STATISTICAL METHODS}

Statistical testing was conducted with the statistical package for the social science system version SPSS version 17.0. Continuous variables were presented as mean $\pm S D$, and categorical variables were presented as absolute numbers and percentage. The comparison of normally distributed continuous variables between the groups was performed using Student's t-test. Nominal categorical data between the groups were compared using Chi-square test or Fisher's exact test as appropriate. $\mathrm{P}<0.05$ was considered statistically significant.

\section{RESULTS}

This work included two groups of patients: Control Group which included 80 normal hearing Ears (46 Males and 34 Females) with no audio logical complaints and Study Group included 80 Ears (46 Males and 34 Females) complaining of tinnitus. The mean ages of subjects in control and study group were $30.64 \pm 10.39$ years and $33.41 \pm 11.27$ years respectively. Tinnitus was bilateral in 28 subjects $(53.84 \%)$ and unilateral in 24 subjects (46.16\%). The mean tinnitus duration was $6.47 \pm 8.37$ months. Both control and study group showed significant difference in TEOAE and DPOAE study. In TEOAE, 8 $(10 \%)$ ears in control group and 30 ears $(37.5 \%)$ in study group showed test result as REFER whereas in DPOAE 10 (12.5\%) ears in control group and 35 (43.8\%) ears showed test result as REFER. All these result were statistically significant with $p$ value $<0.001$ (Table 1 ). Absolute wave latencies and Inter Peak latencies of BERA between the two groups were compared. There was significant difference in absolute latency of WAVE I with $p$ value 0.031 while difference between all other parameters in the two groups were insignificant as shown in Table 2. Results of BERA were also compared between ears which were REFER or PASS in OAE in control group and it was observed that the wave I was significantly prolonged in REFER group with $p$ value 0.025 and IPL I-V significantly decreased ( $p$ value 0.004 ) (Table 3 ). There

Table 1. TEOAE and DPOAE Findings.

\begin{tabular}{|c|c|c|c|c|c|c|}
\hline & & \multicolumn{4}{|c|}{ Groups } & \multirow{3}{*}{ P Value } \\
\hline & & \multicolumn{2}{|c|}{ Control Group } & \multicolumn{2}{|c|}{ Study Group } & \\
\hline & & No. of EARS & $\%$ & No. of EARS & $\%$ & \\
\hline & Pass & 72 & $90.00 \%$ & 50 & $62.50 \%$ & \\
\hline \multirow[t]{2}{*}{ TEOAE } & Refer & 8 & $10.00 \%$ & 30 & $37.50 \%$ & $<0.001$ \\
\hline & Pass & 70 & $87.50 \%$ & 45 & $56.30 \%$ & \\
\hline DPOAE & Refer & 10 & $12.50 \%$ & 35 & $43.80 \%$ & $<0.001$ \\
\hline
\end{tabular}

Table 2. BERA - Absolute Wave Latencies and Inter-Peak Latency.

\begin{tabular}{ccc}
\hline Content & Control Group $(\mathbf{n}=\mathbf{8 0})(\mathbf{m s e c})$ & Study Group $(\mathbf{n}=\mathbf{8 0})(\mathbf{m s e c})$ \\
\cline { 2 - 3 } & Mean \pm SD & Mean \pm SD \\
\hline WAVE- I & $1.78 \pm 0.29$ & $1.88 \pm 0.27$ \\
WAVE - III & $3.77 \pm 0.29$ & $3.80 \pm 0.28$ \\
WAVE - V & $5.68 \pm 0.32$ & $5.74 \pm 0.23$ \\
IPL- I - III & $1.99 \pm 0.26$ & $1.92 \pm 0.24$ \\
IPL- III - V & $1.95 \pm 0.19$ & $1.94 \pm 0.16$ \\
IPL-I-V & $3.94 \pm 0.26$ & $3.91 \pm 0.33$ \\
\hline
\end{tabular}

Table 3. Relation between TEOAE and BERA in control group.

\begin{tabular}{cccc}
\hline & \multicolumn{2}{c}{ TEOAE - Control Group (msec) } & \multirow{2}{*}{ P Value } \\
\cline { 2 - 3 } & PASS $(\mathbf{n = 7 2 )}$ & REFER (n=8) & \\
\cline { 2 - 3 } & Mean \pm SD & Mean \pm SD & 0.025 \\
WAVE- I & $1.75 \pm 0.29$ & $1.99 \pm 0.19$ & 0.576 \\
WAVE - III & $3.76 \pm 0.29$ & $3.82 \pm 0.25$ & 0.934 \\
WAVE-V & $5.68 \pm 0.33$ & $5.67 \pm 0.26$ & 0.068 \\
IPL- I- III & $2.01 \pm 0.27$ & $1.83 \pm 0.13$ & 0.115 \\
IPL- III - V & $1.96 \pm 0.19$ & $1.85 \pm 0.13$ & 0.004 \\
IPL-I-V & $3.96 \pm 0.26$ & $3.68 \pm 0.11$ & \\
\hline
\end{tabular}


was no significant difference seen in between PASS and REFER group in BERA outcomes in study group (Table 4). All BERA waves in patients with DPOAE as REFER were prolonged in both control and study group, though the results were not statistically significant, except for decreased IPL III-V in study group (Table 5). No significant difference was seen in BERA parameters in control group patients with DPOAE result as either PASS or REFER (Table 5). In Table 6, IPL-III-V was significantly reduced in patients with DPOAE result as REFER in study group. Table 7 shows, Patients with DPOAE as PASS shows a significant reduction in IPL-I -III in study group as compared to control group ( $p$ value 0.377 ). There is no significant difference in BERA parameters between study or control group in patients with DPOAE and TEOAE result as REFER (Tables 8 and 10). Wave I was significantly prolonged along with reduction in IPL- I-III in study group in patients with TEOAE as PASS (Table 9).

\section{DISCUSSION}

Tinnitus is a common and persistent symptom. The pathogenesis and site of origin have yet to be clearly

Table 4. Relation between TEOAE and BERA in study group.

\begin{tabular}{ccc}
\hline & \multicolumn{2}{c}{ TEOAE - Study Group (msec) } \\
\cline { 2 - 3 } & PASS (n=50) & REFER (n=30) \\
\cline { 2 - 3 } & Mean \pm SD & Mean \pm SD \\
\hline WAVE- I & $1.89 \pm 0.23$ & $1.86 \pm 0.33$ \\
WAVE - III & $3.81 \pm 0.31$ & $3.78 \pm 0.22$ \\
WAVE-V & $5.76 \pm 0.24$ & $5.71 \pm 0.21$ \\
IPL- I- III & $1.92 \pm 0.19$ & $1.92 \pm 0.30$ \\
IPL- III - V & $1.95 \pm 0.17$ & $1.93 \pm 1.16$ \\
IPL-I-V & $3.92 \pm 0.27$ & $3.90 \pm 0.40$ \\
\hline
\end{tabular}

Table 5. Relation between DPOAE and BERA in Control group.

\begin{tabular}{cccc}
\hline & \multicolumn{2}{c}{ DPOAE - Control Group (msec) } & \multirow{2}{*}{ P Value } \\
\cline { 2 - 3 } & PASS $(\mathbf{n}=\mathbf{7 0})$ & REFER $(\mathbf{n}=10)$ & \\
\cline { 2 - 3 } & Mean \pm SD & $1.91 \pm 0.27$ & 0.127 \\
WAVE- I & $1.76 \pm 0.29$ & $3.84 \pm 0.27$ & 0.413 \\
WAVE - III & $3.76 \pm 0.29$ & $5.73 \pm 0.23$ & 0.581 \\
WAVE-V & $5.67 \pm 0.33$ & $1.93 \pm 0.14$ & 0.441 \\
IPL- I - III & $2.00 \pm 0.28$ & $1.89 \pm 0.16$ & 0.345 \\
IPL- III - V & $1.95 \pm 0.19$ & $3.82 \pm 0.19$ & 0.146 \\
IPL-I-V & $3.95 \pm 0.27$ & \\
\hline
\end{tabular}

Table 6. Relation between DPOAE and BERA in study group.

\begin{tabular}{cccc}
\hline & \multicolumn{2}{c}{ DPOAE - Study Group } & \multirow{2}{*}{ P Value } \\
\cline { 2 - 3 } & PASS $(\mathbf{n = 4 5 )}$ & REFER $(\mathbf{n}=\mathbf{3 5})$ & Mean \pm SD \\
\cline { 2 - 3 } & Mean \pm SD & $1.90 \pm 0.33$ & 0.518 \\
WAVE- I & $1.86 \pm 0.22$ & $3.85 \pm 0.26$ & 0.114 \\
WAVE - III & $3.75 \pm 0.29$ & $5.75 \pm 0.24$ & 0.706 \\
WAVE-V & $5.73 \pm 0.23$ & $1.96 \pm 0.28$ & 0.258 \\
IPL- I - III & $1.90 \pm 0.19$ & $1.90 \pm 0.15$ & 0.031 \\
IPL- III - V & $1.98 \pm 0.17$ & $3.90 \pm 0.37$ & 0.787 \\
IPL-I-V & $3.92 \pm 0.29$ & & \\
\hline
\end{tabular}

Table 7. Relation between BERA in study and control group with DPOAE as 'PASS'

\begin{tabular}{|c|c|c|c|}
\hline & \multicolumn{2}{|c|}{ DPOAE } & \\
\hline & PASS $(n=70)$ Control Group & PASS $(n=45)$ Study Group & \\
\hline & Mean \pm SD & Mean \pm SD & \\
\hline WAVE- I & $1.76 \pm 0.29$ & $1.86 \pm 0.22$ & 0.507 \\
\hline WAVE - III & $3.76 \pm 0.29$ & $3.75 \pm 0.29$ & 0.857 \\
\hline WAVE-V & $5.67 \pm 0.33$ & $5.73 \pm 0.23$ & 0.289 \\
\hline IPL- I - III & $2.00 \pm 0.28$ & $1.90 \pm 0.19$ & 0.037 \\
\hline IPL- III - V & $1.95 \pm 0.19$ & $1.98 \pm 0.17$ & 0.391 \\
\hline IPL-I-V & $3.95 \pm 0.27$ & $3.92 \pm 0.29$ & 0.573 \\
\hline
\end{tabular}


Table 8. Relation between BERA in study and control group with DPOAE AS 'REFER'.

DPOAE

\begin{tabular}{cccc} 
& REFER $(\mathbf{n}=\mathbf{1 0})$ Control Group & REFER $(\mathbf{n}=\mathbf{3 5})$ Study Group & Value \\
\cline { 2 - 4 } & Mean $\pm \mathbf{S D}$ & Mean \pm SD & 0.915 \\
WAVE- I & $1.91 \pm 0.27$ & $1.90 \pm 0.33$ & 0.915 \\
WAVE - III & $3.84 \pm 0.27$ & $3.85 \pm 0.26$ & 0.815 \\
WAVE-V & $5.73 \pm 0.23$ & $5.75 \pm 0.24$ & 0.726 \\
IPL- I - III & $1.93 \pm 0.14$ & $1.96 \pm 0.28$ & 0.855 \\
IPL- III - V & $1.89 \pm 0.16$ & $1.90 \pm 0.15$ & 0.515 \\
IPL-I-V & $3.82 \pm 0.19$ & $3.90 \pm 0.37$ &
\end{tabular}

Table 9. Relation between BERA in study and control group with TEOAE as 'PASS'.

\begin{tabular}{cccc}
\hline & \multicolumn{3}{c}{ TEOAE } \\
\cline { 2 - 4 } & PASS $(\mathbf{n}=\mathbf{7 2})$ Control group & PASS $(\mathbf{n = 5 0 )}$ Sudy Group & Value \\
\cline { 2 - 4 } & Mean \pm SD & Mean \pm SD & 0.005 \\
WAVE- I & $1.75 \pm 0.29$ & $1.89 \pm 0.23$ & 0.364 \\
WAVE - III & $3.76 \pm 0.29$ & $3.81 \pm 0.31$ & 0.145 \\
WAVE-V & $5.68 \pm 0.33$ & $5.76 \pm 0.24$ & 0.044 \\
IPL- I - III & $2.01 \pm 0.27$ & $1.92 \pm 0.19$ & 0.766 \\
IPL- III - V & $1.96 \pm 0.19$ & $1.95 \pm 0.17$ & 0.412 \\
IPL-I-V & $3.96 \pm 0.26$ & $3.92 \pm 0.27$ & \\
\hline
\end{tabular}

Table 10. Relation between BERA in study and control group with TEOAE as 'REFER'.

\begin{tabular}{cccc}
\hline & \multicolumn{3}{c}{ TEOAE } \\
\cline { 2 - 4 } & Mean \pm SD & Mealue \\
\cline { 2 - 4 } & $1.99 \pm 0.19$ & $1.86 \pm 0.33$ & 0.295 \\
WAVE- I & $3.82 \pm 0.25$ & $3.78 \pm 0.22$ & 0.659 \\
WAVE - III & $5.67 \pm 0.26$ & $5.71 \pm 0.21$ & 0.368 \\
WAVE-V & $1.83 \pm 0.13$ & $1.92 \pm 0.30$ & 0.416 \\
IPL- I - III & $1.85 \pm 0.13$ & $1.93 \pm 1.16$ & 0.848 \\
IPL- III - V & $3.68 \pm 0.11$ & $3.90 \pm 0.40$ & 0.135 \\
IPL-I-V & & REFER $(\mathbf{n}=\mathbf{3 0})$ Study Group & \\
\hline
\end{tabular}

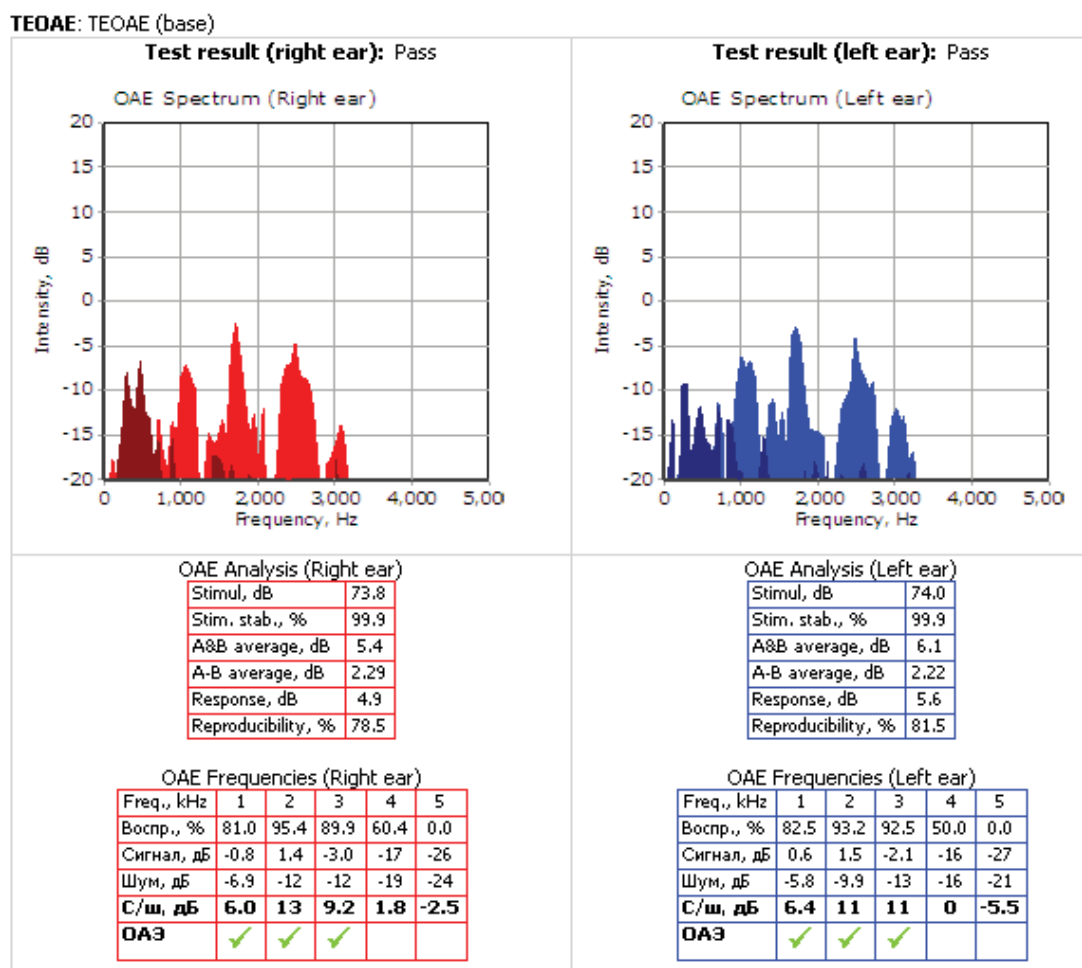

Figure 4. Figure showing the TEOAE TRACINGS 
established. Tinnitus is often a feature of primary ear disease usually associated with hearing loss, but it may also occur in patients with normal hearing. This study focused on the latter group. The lack of scientific evidence to prove that tinnitus arises from cochlear damage in normal-hearing patients has encouraged us to investigate whether patients with tinnitus show changes in the auditory pathways. In a study by Laskar et al. ${ }^{8}$ concluded that all age groups can be affected by tinnitus with middle age group and above had a higher incidence. Most common age group affected was 41-50 years followed by 31-40 years ${ }^{7}$. In our study the mean age of individuals in study group was $33.41 \pm 11.27$ years. The reason for lower mean age is that we excluded the individuals above age of 55 years, in order to eliminate component of prebycusis. Slight male predominance was found in our study with $57.75 \%$ males and $42.25 \%$ females. Yenigun reported that males were more likely to get affected by tinnitus whereas there was female prediliction seen in other studies by the authors ${ }^{(7-11)}$. Tinnitus can affect unilaterally or bilaterally with slight left ear predominance among patients with unilateral tinnitus. Laskar found bilateral complaint in $50 \%$, isolated left ear involvement in $27.2 \%$ and right ear involvement in $22.2 \%$ patients ${ }^{7}$. Tsai in his work on audiometric asymmetry and tinnitus laterality also encountered left ear preponderance with $38.70 \%$ patient's tinnitus laterality to left ear and $28.22 \%$ patients with laterality to right ear ${ }^{8-12}$. Comparable observations regarding the laterality were seen in our study with bilateral presentation in $52.84 \%$ patients, and unilateral isolated complain in left and right ear in $26.92 \%$ and $19.93 \%$ respectively. Recent studies have found that instead of outer hair cells, the synapses between inner hair cells and auditory nerve fibers, especially low spontaneous rate nerve fibers, are most vulnerable to the effects of noise, aging, and ototoxic medication. The damage of synapses causes sound-coding deficits in temporal and intensity processing leading to perceptual hearing difficulties, but cannot be detected by current standard hearing diagnostic procedures (1225). In our study we have not accounted the possibility of hidden hearing loss caused due to noise exposure. This prospect is discussed in recent work by Liberman theories of cochlear synaptopathy. In a review on animal models on noise induced and age-related hearing loss by Liberman and Kujawa, they proposed that well before overt hearing loss there is permanent interruption of synaptic communication between sensory inner hair cells and subsets of cochlear nerve fibers. The silencing of affected neurons alters auditory information processing leading to abnormalities like speech-in-noise difficulties, tinnitus and hyperacusis ${ }^{26}$.

The traditional view of degeneration in sensorineural hearing loss with primary target as hair cells and secondary target as cochlear nerve has been challenged by recent works on mouse and guinea pig. Kujawa and
Liberman concluded that in noise-induced hearing loss, exposures cause only reversible threshold shifts (and no hair cell loss) nevertheless cause permanent loss of $>50 \%$ of cochlear-nerve/hair-cell synapses ${ }^{27}$. Hidden hearing loss could not be identified by conventional pure tone audiometry or speech audiometry. Tone in-noise thresholds may provide frequency specific information in auditory neuropathy patients with normal pure tone threshold. Ralli et al. ${ }^{29}$ found that, despite having normal hearing (thresholds $\leq 25 \mathrm{~dB} \mathrm{HL}$ ), the Old patients had significantly worse tone in-noise thresholds than the Young patients thus concluding the significance of tone in-noise detection as a effective way for diagnosis of hidden hearing loss.28 We observed TEOAE and DPOAE result as REFER in $37.5 \%$ and $43.8 \%$ of patients of tinnitus with normal hearing respectively. Abnormal OAE results may be the indicator of subtle cochlear damage before the abnormality affects hearing sensitivity and appears on the audiogram. Higher prevalence of abnormal outcomes was seen in a study by Granjeiro involving normal hearing patients with abnormal outcomes of TEOAE and DPOAE in $70.2 \%$ and $68.4 \%$ of patients among the tinnitus group.13 Other studies by Ami, Wan, the authors also had abnormal outcomes of OAE testing in patients complaining of tinnitus ${ }^{(13-18)}$.

In our study it was observed that in the subjects of tinnitus with normal hearing, $62.5 \%$ and $56.3 \%$ had normal TEOAE and DPOAE results respectively. These findings suggest that the $\mathrm{OHC}$ dysfunction is not necessary for tinnitus development. On the other hand, the $\mathrm{OHC}$ dysfunction is not sufficient in itself to cause the symptom, because $10-12 \%$ of individuals with $\mathrm{OHC}$ dysfunction (abnormal OAE results) did not complain about tinnitus. Serra et al. concluded in a review on tinnitus patients that, it is important to consider the hypothesis of the involvement of the efferent system in the generation of tinnitus as outer hair cell dysfunction is not necessary for tinnitus development ${ }^{19}$. BERA was done to evaluate the integrity of the auditory pathway from cochlear nerve to the brainstem. We evaluated absolute latencies of wave I, III \& V along with their inter-peak latencies. In our study all absolute latencies were prolonged with a significant difference in absolute latency in wave I between the study and control group. In study by Maurizi et al. and Lemaire only wave I and V were significantly prolonged whereas three waves (I, III and V) were significantly prolonged in works by Beutter, Kehrle and Kumar(20-23). Takwa and Shadman found marginal abnormalities in absolute latencies of all 3 waves, but the results were statistically insignificant ${ }^{(3-5)}$. In contrast to our work, Barnea et al. found normal $A B R$ results in all 17 patients with tinnitus enrolled in their study compared with 19 patients without tinnitus ${ }^{24}$. McKee and Stephens reported normal ABR latencies in all 18 normal hearing patients with tinnitus in their study ${ }^{25}$. The method used in our study to evaluate cochlear status (conventional audiogram) might not have been 
sufficiently sensitive for assessing all aspects of cochlear functioning. This lies in the fact that many commonly encountered hearing losses initially affect the extended high frequencies (above $8 \mathrm{kHz}$ ) that were not evaluated in this study ${ }^{(25-27)}$. OAE and BERA results in normal hearing tinnitus patients are different from one subject to another. Some cases have normal response while others have prolonged absolute latencies or reduced IPLs. This suggests impaired neural firing synchronization and transmission in the auditory pathways in tinnitus patients. These findings also suggested that the pathology underlying tinnitus is not the same in every case with possible brainstem involvement in some cases $^{28}$. This is very important for designing the proper management program and selecting the appropriate medication and instrumentation to relief tinnitus. It also will help to decide the aim of rehabilitation program whether it should be designed to restore the homeostasis of brain activity or to act at more peripheral level. Thus, OAE and BERA might contribute to the work up of these patients and should be performed routinely in tinnitus sufferers. Further evaluation of tinnitus patients based on tinnitus severity, duration and character are recommended to provide more understanding of tinnitus problems and tailoring appropriate rehabilitation programs ${ }^{29}$

\section{LIMITS OF THE STUDY}

The present study has a limitation of small number of patients. The detailed history about noise exposure and ruling out of possibility of undiagnosed hidden hearing loss may have provided better understanding about the electrophysiology of tinnitus

\section{CONCLUSION}

On evaluation of Otoacoustic emissions and Brainstem evoked response audiometry in tinnitus patients with normal hearing, the following conclusions were drawn:

- Otoacoustic emissions (TEOAE and DPOAE) are abnormal ('REFER') in significant number of patients of tinnitus with normal hearing.

- Absolute latency of wave I is significantly prolonged in patients of tinnitus with normal hearing.

- Absolute latencies of Wave III and V are also prolonged, though not significantly.

- Oto Acoustic Emissions (OAE) and Brainstem Evoked Response Audiometry (BERA) should be included in the test battery for the screening of patients complaining of tinnitus even with normal hearing.

- Continued studies with large sample size may lead to development of new treatment and rehabilitation protocols in future.

\section{CONFLICT OF INTEREST}

The Author declares no conflict of interest

\section{REFERENCES}

1. Wan I, Fei Z, Deepak R, Mohammed A. Measurement of subtle auditory deficit in tinnitus patients with normal audiometric thresholds using evoked Otoacoustic emissions and threshold equalizing noise tests. Int Tinnitus J. 2013;18(1):35-44.

2. Datt M, Katarakar A, Alam N, Jain A, Shah P. Relation of distortion product otoacoustic emission and tinnitus in normal hearing patients: A pilot study. J Noise Health. 2014;16:69-72.

3. Shadman N, Ali F, Rasool P, Masoomeh P. Cochlear and Brainstem Audiologic Findings in Normal hearing tinnitus Subjects in Comparison with non-tinnitus Control Group. Acta Medica Iranica. 2014;51(11):822-6.

4. GA Van Z, Lionel C, Kris H, David K, Rudolf P. Symposium Otoacoustic emissions. Int J Ped Otorhinolaryngology.1995;32(Suppl.):S213-16.

5. Takwa AG. Auditory brainstem response audiometry in tinnitus patients. Egyptian $\mathrm{J}$ of Ear, Nose, Throat and Allied Sciences. 2011;12:115-20.

6. Helga K, Ronaldo G, Andre S, Roberta B, Vanessa A, carlos O. Comparison of

7. Auditory Brainstem Response Results in Normal-Hearing Patients With and Without Tinnitus. Arch Otolaryngol Head Neck Surg. 2008;134(6):647-51.

8. Laskar HA, Shunyu NB, Medhi J, Jamil K, Nongiej C, Khyriem E. Tinnitus: A hospital-based retrospective study. Ind J Otol. July 2015;21(3):197-200

9. Alper Y, Remzi D, Fadlullah A, Servet A, Hakan D. Assessment of tinnitus with tinnitus severity index, tinnitus handicap inventory and distortion product otoacoustic emissions in patients with normal hearing and hearing loss. Kulak Burun Bogaz Ihtis Derg. 2014;24(1):11-16.

10.Josef S, Gary C, Wildon F. Prevalence and Characteristics of Tinnitus among US Adults. Am J Med. August 2010;123(8):711-18.

11. Yildimir G, Berkiten G, kuzdere M, Ugras H. High frequency audiometry in patients presenting with Tinnitus. J Int Adv Otology. 2010;6(3):401-7.

12.Sujoy M, Anirban B, Pragati S. The Impact of Tinnitus on Sufferers in Indian Population. Indian J Otolaryngol Head Neck Surg.2014;66(Suppl1):37-51.

13.Tsai BS, Sweetow RW, Cheung SW. Audiometric Asymmetry and Tinnitus Laterality. The Laryngoscope.May 2012;122:1148-53.

14.Bezerra RL, Almeida VF, Andre LL, Oliveira CA. Transient and distortion product evoked oto-acoustic emissions in normal hearing patients with and without tinnitus. Otolaryngol Head Neck Surg.2008;138(4):502-6.

15.Gentil F, Meireles S, Roza T, Santos C, Parente M, Almeida E, et al. Comparison of otoacoustic emissions in patients with tinnitus having normal hearing versus mild hearing loss. Int Tinnitus J. 2015; 19(2):39-46.

16. Fernandes LD, Santos TM. Tinnitus and normal hearing: a study on the transient Otoacoustic emissions suppression Braz J otorhinolaryngol. 2009;75(3):414-9.

17. Qasem H, Assaf S, Jamous NA, Hroot A, Tubish K, Husban A, et al. Otoacoustic Emissions and tinnitus in normal hearing. $J$ of Royal Medical Sevices. 2010;17(2):27-31.

18. Ektor O, Yotaka F, Fabio S. Distortion product Otoacousticemmisions in tinnitus patients. Int Tinnitus J. 2004;10(1):13-16.

19.Ami M, Abdullah A, Awang M, Liyan B, Saim L. Relation of Distortion Product Otoacoustic emission with tinnitus. Laryngoscope. April 2008;118:712-17.

20.Serra L, Novanta G, Sampaio AL, Oliveira CA, Granjeiro R, Braga SC. The Study of Otoacoustic Emissions and the Suppression 
of Otoacoustic Emissions in Subjects with Tinnitus and Normal Hearing: An Insight to Tinnitus Etiology. Int Arch Otorhinolaryngol. 2015;19:171-5

21. Maurizi M, Ottaviani F, Paludetti G, Almadori G, Tassoni A. Contribution to the differentiation of peripheral versus Central Tinntius via Auditory Brainstem Response Evaluation. Audiology.1985;24:207-16.

22.Lemaire MC, Beutter P. Brainstem Auditory Evoked Responses in Patients with Tinnitus. Audiology. 1995 Nov-Dec;34(6):287-300.

23. Kumar GR, Murthy VA. A study of brainstem Auditory evoked responses in Normal hearing patients with Tinnitus. Indian J Otolaryngol Head Neck Surg. 2016;68(4):429-33.

24. Barnea G, Attias J, Shahar A. Tinnitus with Normal Hearing Sensitivity: Extended High-Frequency Audiometry and Auditory-Nerve BrainStem-Evoked Responses. Audiology.1990;29(1):36-45.
25. Mckee GJ, Stephens SD. An Investigation of Normally Hearing Subjects with Tinnitus. Audiology.1992;31(6):313-7.

26.Zheng $Y$, guan J. Cochlear syanptopathy: A review of hidden hearing loss. Journal of Otorhinolaryngol Disorders and Treatments.2018;1(1):138-47.

27.Liberman MC, Kujawa SG. Cochlear synaptopathy in acquired sensorineural hearing loss: Manifestations and mechanisms. Hear Res. 2017;349:138-47.

28. Kujawa SG, Liberman MC. Synaptopathy in the noise-exposed and aging cochlea: Primary neural degeneration in acquired sensorineural hearing loss. Hear Res.2015;330:191-9.

29. Ralli M, Greco A, De Vincentiis M, Sheppard A, Cappelli G, Neri I, et al. Tone-in-noise detection deficits in elderly patients with clinically normal hearing. Am J Otolaryngol. 2019;40(1):1-9. 